Article

\title{
Hedging on Betting Markets
}

\author{
Gustav Axén ${ }^{1, *} \mathbb{D}$ and Dominic Cortis ${ }^{2}$ (D) \\ 1 Private Practice, 11737 Stockholm, Sweden \\ 2 Department of Insurance, FEMA, University of Malta, Msida, 2080 MSD, Malta; dominic.cortis@um.edu.mt \\ * Correspondence: gu.axen@gmail.com
}

Received: 3 July 2020; Accepted: 29 July 2020; Published: 25 August 2020

check for updates

\begin{abstract}
The possibility to use hedging strategies is an often neglected aspect in the literature on prediction/betting markets, as most papers assume that bettors will bet according to their beliefs about the probability of the outcome of the event, as opposed to the direction in which the odds will move. This ignores strategies that try to buy low and sell high through exploiting price changes, which is an important aspect to incorporate to fully understand market pricing. In this paper, we derive the key mathematical results in using hedging strategies through taking opposite positions to an initial bet after the market odds have changed and show that a profit can be made without explicitly speculating on the probability of the outcomes. We also discuss two sources of inefficiency that can arise when using hedging strategies in practice: (i) the need to pay a fee when using a betting exchange and (ii) the lack of a lay option (the possibility to bet against outcomes) on some markets, and we analyze how they affect the possibilities to hedge. Many of the results have interesting properties when expressed in terms of the naive probabilities implied by the odds.
\end{abstract}

Keywords: betting markets; Dutching; hedging; betting exchange; betting risk; cash out; lay odds; odds; betting

\section{Introduction}

Betting on the possible outcomes of future events forms a useful information aggregation mechanism, as the price on a betting contract is dependent on the expected probability that it pays off (Manski 2006; Stark and Cortis 2017; Wolfers and Zitzewitz 2006). This makes betting markets useful for academics, as they can be used to predict which scientific results will replicate (Dreber et al. 2015) and serve as inputs to economic models that require proxies for probabilities for their identification strategy (Coulomb and Sangnier 2014). Empirical studies show that betting markets often are accurate in predicting the outcomes of future events and frequently do better than other forecasting methods (Arrow et al. 2008; Berg et al. 2008; Kovalchik 2016).

However, several important aspects of betting are often ignored in the assumptions of the models presented in the academic literature. One such aspect is the possibility of using hedging strategies. While the most straightforward manner to make money on a betting market is to accurately predict the outcome of the event and place money accordingly, it is also possible to make profits through abusing changes in the market odds, just like it is possible to make a profit on other financial instruments by buying low and selling high.

Simply put, a bet can be hedged on the market by taking another bet in the opposite direction with a stake calculated such that the two bets have the same cash flow regardless of the outcome. The previously risky position is then closed, and the position is converted to a future risk-free cash flow (which can be higher or lower than the sum of the stakes of the two bets). This paper serves to introduce the general results and the formal terminology for those positions. While the concept of hedges in betting has been explored in some other contexts in the literature, it has mostly 
been done in order to test behaviorally whether people will detect hedging opportunities, with the greater purpose of testing assumptions about rationality and risk aversion (Frederick et al. 2018, Chatterjee and Mookherjee 2018). Though Newall and Cortis (2019) discussed the possibilities of hedging in a similar manner done here and provided numerical examples, we are not aware of any previous work that covers this generally and derives the key results, which serves as the contribution of this paper. Additionally, we derive the profit when there is no option to lay on the market (i.e., when the bettors do not have the explicit option of betting against an outcome occurring and have to replicate this outcome through combinations of other bets) which, to our knowledge, is a novel topic.

\section{Terminology and Previous Results}

Betting markets can be organized in several ways. Most betting sites use a posted set of odds, which describe to what extent the stake is multiplied if a bet is placed and the corresponding outcome occurs. Another common form is the prediction market format, where people trade contracts that pay a fixed amount if an outcome is realized (Agrawal et al. 2010) ${ }^{1}$. We will use the notation of the former market in order to preserve generality (as prediction market contracts generally cover only two possible outcomes, and we want to cover an arbitrarily large amount). Hence, we consider betting on a set of mutually exclusive and collectively exhausting outcomes of an event, indexed by $N=\{1,2, \ldots, n\}$ with $|N|=n \geq 2 .^{2}$

Betting that $i \in N$ will occur is called to back $i$, and we denote the ordered set of back odds in European/decimal format ${ }^{3}$ as $\alpha=\left(\alpha_{1}, \alpha_{2}, \ldots, \alpha_{n}\right)$. This means that if a back bet on $i$ is placed and then won, the bettor obtains a cash flow of $\alpha_{i}$ times the stake, for a total return of investment of $\alpha_{i}-1$ times the stake (as is standard in the literature, we ignore discounting for simplicity (Chen 2005), as most betting takes place in a relatively short time span). This means that it must hold that $\alpha_{i}>1, \forall i \in N$, or the return from a back bet will never be positive. For instance, if $\alpha_{i}=2$, a bettor gets back double his ${ }^{4}$ investment if $i$ occurs, making a return of $100 \%$, and loses the stake if any outcome except for $i$ occurs.

Therefore, in order for the bet to have an expected return of zero, the probability of the outcome needs to be $\frac{1}{\alpha_{i}}$, which gives the naive intuition that the decimal form odds can be interpreted as probabilities. However, the interpretation of odds as probabilities is confounded by multiple factors. As is discussed below, the probabilities do not necessarily add up to $100 \%$, and this interpretation additionally reflects the added assumption of risk-neutrality, which can be questioned. Nevertheless, this naive probability interpretation has uses and interesting implications, as we will see below.

Some market forms also allow bettors to place bets against outcomes occurring, which is called laying an outcome. In other words, a lay bet on outcome $i$ pays off if $i$ does not occur, equivalent to any $j \neq i \in N$ occurring. A lay position is the inverse of a back position, which means that higher odds result in higher risks rather than higher potential returns. To lay a certain amount is called to risk the amount that is bet by the lay bettor, to contrast it with the back odds.

The lay odds need not be the same as the back odds, and we therefore separately define the ordered set of lay odds as $\beta=\left(\beta_{1}, \beta_{2}, \ldots, \beta_{n}\right)$. The cash flow on which the lay bet on $i$ is won is defined as $\frac{\beta_{i}}{\beta_{i}-1}$ multiplied by the amount risked, for a winning return of $\frac{\beta_{i}}{\beta_{i}-1}-1=\frac{1}{\beta_{i}-1}$. The reason for the difference in notation is that $\beta_{i}$ corresponds to the back odds that a lay bettor would offer someone taking a back bet on the same outcome such that their positions cancel out, and the function

1 Another possible method is the parimutuel method, where the odds are determined only after people have bet. This is however a different problem as the bettors are unaware of their expected return when placing bets (Ottaviani and Sørensen 2005, 2009).

2 We approximately follow the notation of Axén and Cortis (2019) here as the paper studies a similar problem using the same inputs.

3 See Cortis (2015) for alternative ways to denote odds.

4 We tossed a coin and use the male pronoun throughout the paper. 
for cash flow is derived from that. ${ }^{5}$ Again, $\beta_{i}>1, \forall i \in N$ is a necessary condition in order for any lay position to be able to make a potential profit.

Using this notation, we define $\pi(\boldsymbol{\alpha}) \equiv \sum_{i \in N} \frac{1}{\alpha_{i}}-1$ as the back overround, $\pi(\boldsymbol{\beta}) \equiv \sum_{i \in N} \frac{1}{\beta_{i}}-1$ as the lay overround, and $\pi\left(\alpha_{i}, \beta_{i}\right) \equiv \frac{1}{\alpha_{i}}-\frac{1}{\beta_{i}}$ as the spread overround for $i \in N$, following Axén and Cortis (2019). These are measures of how high the odds are on the market as a whole, and these can be interpreted as the excess naive probabilities that arise from market inefficiencies. For example, if the back overround is $5 \%$, this means the naive probabilities of the decimal odds for the back odds add up to $105 \%$. The overrounds generally differ from zero due to frictions in the market, for example due to limited liquidity (see Flepp et al. 2017), because bookmakers offer less than optimal odds in order to be able to make a profit (see Franck et al. 2010), or because of the so-called favorite-long shot bias, which is the empirical observation that betting market odds sometimes overestimate the likelihood of outcomes with a low probability occurring (see Buhagiar et al. 2018; Cain et al. 2000; Candila and Scognamillo 2018). We analyze the implications of this for our results further below.

Several important results on market pricing are expressed in terms of the overrounds. If the prices on the betting market follow even the most elementary assumptions about economic rationality, they are constrained by the fact that (i) they should not allow for arbitrage (Cortis 2015) and that (ii) no bet should be strictly dominated by some combination of other bets, as this would render a bet useless for any bettor regardless of preferences and beliefs (Axén and Cortis 2019).

Example 1. For a simple example when there is arbitrage, consider an event with $n=2$ possible outcomes and the back odds $\boldsymbol{\alpha}=(3,3)$. Betting $\$ 1$ on Outcome 1 and $\$ 1$ on Outcome 2 yields a total $\$ 2$ bet, but the position pays off $\$ 3$ regardless of the outcome, making a guaranteed profit of $\$ 1$. Hence, there is arbitrage.

Example 2. For a simple example where there is a dominated asset available on the market that can be replicated by another asset, consider the case of $n=2$ possible outcomes with $\boldsymbol{\alpha}=(1.5,2)$ and $\boldsymbol{\beta}=(3,2)$. Risking $\$ 1$ taking a lay bet on Outcome 2 gives the payoff $\$ 1 \times \frac{\beta_{2}}{\beta_{2}-1}=\$ 2$ if Outcome 1 occurs and zero else. In other words, the bettor would win $\$ 1$ in total if Outcome 1 occurs (as Outcome 2 did not occur and outcomes are assumed to be mutually exclusive) and lose $\$ 1$ if not. A direct back bet of $\$ 1$ on Outcome 1 pays off $\alpha_{1}=1.5$ if Outcome 1 occurs and zero else. In this case, the bettor would win $\$ 0.5$ in total if Outcome 1 occurs, but lose $\$ 1$ if it does not. Hence, the direct back bet on Outcome 1 is strictly dominated by the lay bet on Outcome 2, and no rational bettor will ever use the direct back bet.

The general case has already been covered in previous work, so we simply state that:

Lemma 1. If it holds that:

$$
\begin{aligned}
\beta_{i} \geq \alpha_{i} \Leftrightarrow \pi\left(\alpha_{i}, \beta_{i}\right) & \geq 0, \forall i \in N \\
\pi(\boldsymbol{\alpha}) & \geq 0 \\
-\pi(\boldsymbol{\beta}) & \geq 0
\end{aligned}
$$

there is no arbitrage on the market that can be exploited by the bettors. If it also holds that:

$$
\begin{aligned}
\pi(\boldsymbol{\alpha}) & \geq \pi\left(\alpha_{i}, \beta_{i}\right), \forall i \in N \\
-\pi(\boldsymbol{\beta}) & \geq \pi\left(\alpha_{i}, \beta_{i}\right), \forall i \in N
\end{aligned}
$$

there does not exist a bet that can be replicated by a portfolio of other bets such that taking it has strictly dominated payoffs for any set of beliefs/strategies.

5 See Axén and Cortis (2019) for the full derivation. 
Proof. See Axén and Cortis (2019).

Another concept that we need to prove our results is Dutching. This means that a stake can be split up into more than one outcome of the event, increasing the probability of winning, but reducing the winnings in each possible case, as the stake is divided between multiple outcomes. By definition, a Dutching portfolio is placed such that all possible outcomes within the portfolio yield the same payoff (Peel 2017).

Example 3. Consider a market with $n=3$ possible outcomes of the event and the back odds $\alpha=(4,4,1.5)$. Taking a Dutching portfolio over Outcomes 1 and 2 means betting half the stake on Outcome 1 and half the stake on Outcome 2. This gives the bettor a return of $\frac{4}{2}-1=100 \%$ if Outcome 1 or Outcome 2 occurs, and he loses the stake if Outcome 3 occurs. The bettor has increased his probability of winning as he bets on two different outcomes, but has given up half of his potential payoff as he splits the stake between them.

As has been shown in previous work, the general case is:

Lemma 2. A Dutching portfolio backing ${ }^{6}$ some $N^{C} \subseteq N$ yields the cash flow of:

$$
\left(\sum_{i \in N^{C}} \frac{1}{\alpha_{i}}\right)^{-1}
$$

times the stake if some $i \in N^{C}$ occurs.

Proof. See Axén and Cortis (2019).

For our results that take place over more than one time period, we use the prime symbol ("'l') to denote the value in the latter time period, so the back odds for $i$ in the later time period is denoted $\alpha_{i}^{\prime}$, the back overround is $\pi\left(\boldsymbol{\alpha}^{\prime}\right)$, and so on, with the values in the former period denoted as above.

Finally, the cash out option merits a brief discussion. In order to accommodate the type of logic presented in this paper, where bettors can take opposite positions to the initial one that close the position, many market makers have started to offer the option to convert an outstanding bet to an instant cash flow, effectively "selling" the bet for an instant payout, which is called cashing out. With a zero interest rate and no limits on liquidity, the fair market value of the cash out option should be equivalent to the guaranteed profit (or loss) that can be made from hedging the bet by taking the opposite position, but this is not necessarily the case in practice due to a positive interest rate and liquidity constraints (Newall and Cortis 2019). In reality, the cash out option should pay out less than the hedging option in order to compensate for obtaining the money earlier (as opposed to waiting until the outcome of the event is resolved), as well as not having to put up the additional liquidity to pay for the other bet. However, those differences cannot be quantified without making a series of assumptions about bettor preferences, which is why it goes beyond the scope of this paper.

\section{The General Case}

The intuition of the manner in which a hedge is placed is simple. A bettor first takes a position in one direction and then bets an additional amount in the other direction, abusing the fact that the odds have changed in such a manner that after taking the second bet, he obtains a guaranteed payoff regardless of the outcome of the event. This means that the risk is eliminated after the second bet has been placed as he has one bet on a given outcome occurring and another bet on that same outcome not occurring.

\footnotetext{
6 An equivalent result can be derived for Dutching over lay bets, but it is not necessary for our purposes.
} 
Example 4. Consider a case where the back odds are equal to the lay odds, that for $i \in N$, we have $\alpha_{i}=\beta_{i}=4$, and that $\$ 1$ is bet backing $i$. This means that when the bet is resolved, the bettor has a cash flow of $\$ 4$ if $i$ occurs and zero if $i$ does not occur. If the odds in the later time period drop such that $\alpha_{i}^{\prime}=\beta_{i}^{\prime}=2$, the bettor can risk $\$ 2$ laying $i$, which gives him a cash flow of $\$ 2 \times \frac{\beta_{i}^{\prime}}{\beta_{i}^{\prime}-1}=\$ 4$ if $i$ does not occur. In other words, he now has a guaranteed cash flow of $\$ 4$ regardless of the outcome of the event. As he has only staked \$3 in total, this means he is guaranteed a profit of $\$ 1$ after closing the position.

This also means that the bettor does not have to explicitly consider the actual probabilities of the outcomes, only the direction in which the odds will move. If it can be predicted that the odds will decrease (increase), as they should do when the probability of the outcome increases (decreases), and this is reflected by the market: a bettor can profit from accurately interpreting that the market has undervalued (overvalued) the probability of the outcome occurring. This means that betting on outcomes can be profitable as long as their probabilities are undervalued (overvalued), but they do not have to be likely (unlikely) to occur in absolute terms in order for such a strategy to be profitable.

We first consider an arbitrary market with both a back and lay option and show the result for the simplest case. We obtain:

Proposition 1. The return made from taking a back position and then closing it through taking a lay bet on the same outcome is:

$$
\frac{\alpha_{i}}{\beta_{i}^{\prime}}-1
$$

which means that a profit is made iff:

$$
\alpha_{i}>\beta_{i}^{\prime}
$$

and the additional liquidity required to close the position when placing the second bet is equal to:

$$
\alpha_{i} /\left(\frac{\beta_{i}^{\prime}}{\beta_{i}^{\prime}-1}\right)
$$

times the original stake.

Proof. First, the bettor bets one unit (normalized) backing $i$, leaving him with a cash flow of $\alpha_{i}$ if $i$ occurs and zero else. In order to close the position, $k>0$ units must be risked on the lay bet such that the cash flow is the same regardless of the outcome of the event, i.e., $\alpha_{i}=\left(\frac{\beta_{i}^{\prime}}{\beta_{i}^{\prime}-1}\right) k \rightarrow k=\alpha_{i}\left(\frac{\beta_{i}^{\prime}-1}{\beta_{i}^{\prime}}\right)$. The total amount bet is $1+\alpha_{i}\left(\frac{\beta_{i}^{\prime}-1}{\beta_{i}^{\prime}}\right)$, and taking the guaranteed cash flow minus the stakes of both of the bets yields the result.

There are several things worth noting about these results that will also hold for many of the subsequent findings. As this represents the most intuitive case, however, we provide some remarks on the results before we proceed.

First, note that we calculate return based on the initial stake and do not take into consideration the additional stake required to close the position as a basis on which the return is calculated. This is because the cash flow of the second bet is guaranteed to be returned, and only the return on the risky capital is interesting for our purposes.

Second, the return expression corresponds exactly to the ratio of the drop in odds, under the assumption that a bet can be backed and laid with the same odds $\left(\alpha_{i}^{\prime}=\beta_{i}^{\prime}\right)$. If this is not the case and $\alpha_{i}^{\prime}<\beta_{i}^{\prime}$, then the odds need to drop more in order for the bettor to make the same profit, as is intuitive. The formula is also equivalent to the standard financial return formula, only written in a different 
format as the odds are written as a multiplicator of the stake and not the price of an asset. ${ }^{7}$ This is intuitive as the bettor first "buys" a bet that pays off if $i$ occurs and then "sells" the same bet, making the return the difference in price.

The most common market form that offers a lay option is a betting exchange, where bettors bet directly against each other such that their positions cancel out. The betting exchange charges a fee on the winnings in order to make a profit, however, and therefore, the formula above needs to be interpreted as the odds after the fee is taken if it is to be applied to such a scenario. We elaborate this in Section 4 and show how the result translates to that setting.

Third, it should be noted that in order to arrive at the expression for when there is a profit from the hedged position, it is not strictly necessary in all situations that the bettor be indifferent to the outcomes, as there might be situations where the bettor can arrive at a guaranteed profit while still having a different cash flow in the two scenarios. However, the closer the position is to a profit of zero for one of the outcomes, the more relative capital the bettor needs to allocate to that outcome, and in the limiting case, the bettor needs to allocate such that it is the same for both outcomes (equivalent to the result for Dutching presented in Axén and Cortis 2019), so our method of assuming a completely hedged position works for finding the minimum profit expression generally.

Fourth, the expression explaining when a profit is made corresponds exactly to the no-arbitrage condition presented in Equation (1), with $\beta_{i}$ switched out for $\beta_{i}^{\prime}$. This is intuitive as the act of hedging a bet to a guaranteed profit can be seen as the problem of finding a portfolio of various odds in multiple time periods that together give a guaranteed positive total cash flow, the same as trying to find "arbitrage" in a single time period.

Fifth, it is worth noting that the stake that can be required to close the position can be very large if the corresponding drop in odds is large. Consider the following example:

Example 5. Consider a simplified setting where the back odds of team $i$ winning a soccer league are $\alpha_{i}=5000$, which later drops to $\alpha_{i}^{\prime}=4$, as was the case of Leicester winning the Premier League 2015/2016, often considered to be one of the greatest upsets in sporting history (Newall and Cortis 2019). Assume for simplicity that the back is equal to the lay, i.e., $\beta_{i}^{\prime}=4$. In order to close the position from betting at the odds $\alpha_{i}=5000$, $\alpha_{i} /\left(\frac{\beta_{i}^{\prime}}{\beta_{i}^{\prime}-1}\right)=5000 /\left(\frac{4}{4-1}\right)=3750$ times the size of the original stake is required.

This means that liquidity constraints are likely to become a major issue for the positions that pay off the most, though in a stylized setting, the bettor can borrow and pay it back without any risk (again, in order to quantify this effect, we would have to make additional assumptions about bettor preferences and interest rates, which goes beyond the scope of the paper.)

As the stake required to close the position will always be the ratio of the potential cash flow if the first position pays off over the potential cash flow of the later position (multiplied by the initial stake), we do not show that formula explicitly in the subsequent results, as it will not bring us further insights beyond the ones presented here.

Finally, we note that the maximum return that can be obtained for any given position that is hedged is $\lim _{\beta_{i} \rightarrow 1^{+}} \frac{\alpha_{i}}{\beta_{i}}-1=\alpha_{i}-1$. This is intuitive as the value of the outstanding bet is increased the lower the lay odds are. When the lay odds approach one, it is the same as the market considering the outstanding back bet to be certainly true to pay off, and then, the return approaches what would have been obtained from placing the initial bet and the prediction coming true. This also means that the initial odds cap the profit and that leverage needs to be used in order to obtain greater payoffs. Similarly, the maximum loss that can be obtained is $\lim _{\beta_{i} \rightarrow \infty} \frac{\alpha_{i}}{\beta_{i}}-1=-1$, i.e., losing the initial stake, with the same logic as above. The equivalence also holds for our subsequent results.

7 We can also frame this as the problem of buying a cash flow of $\$ 1$ if $i$ occurs at the price of $\frac{1}{\alpha_{i}}$ and then selling a cash flow of $\$ 1$ if $i$ occurs at the price of $\frac{1}{\beta_{i}^{\prime}}$, which yields the return of investment as (Price sold)/(Price bought) $-1=\alpha_{i} / \beta_{i}^{\prime}-1$. 
We now show the corresponding result for a lay bet that is closed with a back bet after the odds have changed. The intuition is the same, only that a profit is made if the odds increase as opposed to decrease.

Example 6. Consider a bettor that places a lay bet risking $\$ 1$ at the odds $\beta_{i}=1.25$. This means that he has the payoff $\$ 1 \times \frac{\beta_{i}}{\beta_{i}-1}=\$ 5$ if any outcome except for $i$ occurs. At a later stage, the odds have increased such that $\alpha_{i}^{\prime}=\beta_{i}^{\prime}=5$, and he places another bet of $\$ 1$ backing $i$. He has now bet $\$ 2$ in total, but has a guaranteed cash flow of $\$ 5$ regardless of the outcome, making a guaranteed profit.

The general result is (for our subsequent results when the proofs are analogous to that for Proposition 1, we leave them for the Appendix):

Proposition 2. The return made from taking a lay position and then closing it through taking a back bet on the same outcome is:

$$
\left(1-\frac{1}{\alpha_{i}^{\prime}}\right) /\left(1-\frac{1}{\beta_{i}}\right)-1
$$

which means that a profit is made iff:

$$
\alpha_{i}^{\prime}>\beta_{i}
$$

Proof. See Appendix A.1.

As is intuitive, the expression for when a positive return is made does not change (aside from the time indexes of the back and lay, which are reversed), as the problem still consists of finding odds in multiple time periods, which would have given an arbitrage profit had they existed in the same time period. However, the profit expression changes as the stake needed to cover the position is a function of the initial cash flow, which is written differently for the back and lay bets.

The naive probabilistic interpretation is the same (though with time indexes reversed). If we make the probabilistic assumption that $\frac{1}{\beta_{i}}=1-\frac{1}{\alpha_{i}}$, i.e., that the probability that $i$ does not occur is the same as one minus the probability that it occurs (which, again, need not be the case on the market due to inefficiencies) and that the time indexes are reversed, then Equation (7) simplifies to $\frac{\alpha_{i}^{\prime}}{\beta_{i}}-1$, the same as Equation (4). This means that (i) the odds must move in the opposite direction in order for the position to be profitable, as is intuitive, and that (ii) the probabilistic interpretation is equivalent, but expressed in terms of the initial lay odds and the latter back odds.

\section{Hedging on a Betting Exchange}

While the results above are accurate in terms of a general back and lay market, the most common way of organizing markets with a lay option is through a betting exchange. In this market format, people bet directly against each other such that their positions cancel out, and the betting exchange charges a fee equal to a percentage of the winnings of the bettors in order to make a profit. We therefore translate the results to the betting exchange setting in order to be able to generalize to this setting. As the betting exchange charges a fee, an inefficiency is introduced in the market, which has implications for the profitability of the hedging strategy.

Call the before fee ordered set of back odds $\boldsymbol{a}=\left(a_{1}, a_{2}, \ldots, a_{n}\right)$ and lay odds $\boldsymbol{b}=\left(b_{1}, b_{2}, \ldots, b_{n}\right)$. Assume that the betting exchange fee is the fraction $\tau \in(0,1)$, which is taken from the winnings of the bettors, i.e., the amount that the bettor gets back on top of the amount bet.

Example 7. Assume that a bettor bets $\$ 1$ at the before fee odds of $a_{i}=3$ with the fee $\tau=0.05$ and that the bet is won. He has then won $\$ 2$ on top of his initial stake before the fee. The betting exchange charges a fee of $\tau \times \$ 2=0.05 \times \$ 2=\$ 0.1$, and the money the bettor has won after the fee is $\$ 1.90$. 
We then have the following relation between the before and after fee odds:

Lemma 3. The after fee back odds on the betting exchange are:

$$
\alpha_{i}=1+(1-\tau)\left(a_{i}-1\right)
$$

The after fee lay odds on the betting exchange are:

$$
\frac{\beta_{i}}{\beta_{i}-1}=1+\frac{1-\tau}{b_{i}-1} \Leftrightarrow \beta_{i}=1+\frac{b_{i}-1}{1-\tau}
$$

Proof. For the back bet, the intuition is simple, as $\alpha_{i}=\left(\alpha_{i}-1\right)+1$. The one does not have the fee applied to it, as it represents the original stake, and that is not part of the winnings, and the fee is applied to $\alpha_{i}-1$. For the lay bet, we need a conversion that corresponds to the actual cash flow of $\frac{\beta_{i}}{\beta_{i}-1}$. We obtain:

$$
\frac{\beta_{i}}{\beta_{i}-1}=1+(1-\tau)\left(\frac{b_{i}}{b_{i}-1}-1\right)
$$

which simplifies to the expression in the result.

With this terminology, we are able to show the corresponding hedging result for the betting exchange, given that the betting exchange charges the fee on the won bet when two bets in the opposite directions are taken.

Proposition 3. On a betting exchange that charges a fee on the winning bet, taking a back bet and then closing it through a lay bet is profitable iff:

$$
\frac{a_{i}-1}{b_{i}^{\prime}-1}>\frac{1}{(1-\tau)^{2}}
$$

The expression for when a position through taking a lay bet and then closing it with a back bet is the same except with the time indexes of $a_{i}$ and $b_{i}^{\prime}$ changed to $a_{i}^{\prime}$ and $b_{i}$.

Proof. For the back case, we take Proposition 1 and substitute Equations (9) and (10). For the lay case, we take Proposition 2 and substitute the same equations, giving us the equivalent result. (see Appendix A.2 for the full mathematical derivation)

This means that the inefficiency in the market caused by having the betting exchange fee manifests itself through imposing a cap such that the odds have to drop (rise) a non-zero amount in order for them to be profitable. The probabilistic interpretation, however, does not change despite the difference in expression, as the proposition is the same as Propositions 1 and 2, with only a difference in expression, as we have substituted the odds for the odds after a fee.

However, rather than charging the fee on the winnings of the bet that pays off, the betting exchange may instead charge a fee on the net winnings of the two positions. In this more realistic setting, the betting exchange will charge the fee on the winnings after the stakes of both positions are subtracted (if the profit is positive), meaning that the total amount paid will be different from that above.

Example 8. Consider a bettor on a betting exchange who places a back bet of $\$ 1$ that $i$ will occur at the odds $a_{i}=6$. At a later point, he places a lay bet risking $\$ 3$ at the odds $b_{i}^{\prime}=2$. Excluding the fee, the net profit of the two positions if $i$ occurs and the back bet wins is $\$ 6-\$ 1-\$ 3=\$ 2$, and the betting exchange might apply the fee on this $\$ 2$ rather than on the winning back bet, which would have meant applying the fee on $\$ 5$. 
We now assume that the profit is calculated in this manner and that the bettor who places the hedging position knows about it. Then, the amount bet on the second bet must be calculated taking this into account. We obtain the following result:

Proposition 4. If a betting exchange charges a fee only on the net of the winnings of the back and lay positions if the bettor has taken both sides, a bettor taking a back bet and then closing it through a lay bet is profitable iff:

$$
a_{i}>b_{i}^{\prime}
$$

The expression for when a position through taking a lay bet and then hedging it with a back bet is the same except with the time indexes of $a_{i}$ and $b_{i}^{\prime}$ changed to $a_{i}^{\prime}$ and $b_{i}$.

Proof. Assume that the bettor places a back bet with one unit (normalized) on $i$ at the before fee odds $a_{i}$. At a later point, the bettor places $k>0$ units betting against the outcome occurring. If $i$ occurs, the profit before the fee is $\left(a_{i}-1-k\right)$, of which the betting exchange takes $\tau$, leading to a profit of $(1-\tau)\left(a_{i}-1-k\right)$. If $i$ does not occur, the bettor's profit before the fee is $\frac{b_{i}^{\prime}}{b_{i}^{\prime}-1} k-1-k$. Solving for these expressions being equal yields $k=a_{i}\left(1-\frac{1}{b_{i}^{\prime}}\right)$, and inserting this into the profit expression yields the result. The analogous results hold if a lay bet is taken first and is closed with a back bet.

Note that the fact that $\tau$ does not appear in the equation does not mean that it is irrelevant; the fee is still charged on the winnings. However, the break-even conditions do not change, which leads to $\tau$ being absent in the result.

\section{Hedging without a Lay Option}

While taking a lay bet is the most straightforward way to close a back bet, not all markets offer a lay option. This is the most common form of betting through bookmakers, who offer a set of back odds on which they allow users to bet (e.g., Bwin, Bet365, Pinnacle Sports, William Hill). In other words, bookmakers take the lay position on all outcomes and make a profit by setting $\alpha$ such that $\pi(\boldsymbol{\alpha})>0$. This results in less than optimal odds to the users, which works as the markup for providing the opportunity to bet (Cortis 2015; Levitt 2004). The overround introduces an inefficiency similar to the one caused by the betting exchange fee.

However, even when there is no lay option available on the market, a lay bet can still be "replicated" by back bets (Axén and Cortis 2019). If a Dutching portfolio is taken with back bets over $N \backslash i$, a constant payoff is gained if any outcome but $i$ occurs, just like if a lay bet on $i$ were made. In Proposition 2, we showed such a situation for $n=2$, and as has been demonstrated previously, the analogous holds for larger values of $n$ as well (Axén and Cortis 2019). However, the payoff of the replicating portfolio is not necessarily as good as taking the direct lay bet, unless the market is mispriced. Consider the following example for illustration:

Example 9. Consider a market with $n=3$ possible outcomes and the back odds $\alpha=(1.25,4,4)$. If a bettor places half his stake on Outcome 2 and half his stake on Outcome 3, he will double his initial stake if any of those outcomes occur, which is the same as doubling his stake if Outcome 1 does not occur. This makes his portfolio pay off under exactly the same outcomes as though he had placed a lay bet on Outcome 1 at $\beta_{i}=2$. However, if there is a lay option on the market with $\beta_{i}<2$, then he is better off simply taking a lay position on Outcome 1 since he would risk less than half of his potential winnings, which makes the direct lay bet a strictly dominant option. The opposite applies if $\beta_{i}>2$, as in this case, the direct lay bet is strictly dominated and the market is mispriced as no rational bettor would ever take the lay bet.

We therefore also derive the corresponding hedging result, except we replace the payoff of the lay bet with the payoff of taking a backing Dutching portfolio through placing back bets over $N \backslash i$, to demonstrate how hedging can be performed on markets that lack the lay option. We instantly 
find that additional constraints are necessary in order for this type of betting strategy to be able to be profitable:

Proposition 5. In order for any hedging strategy that uses a replicating back bet through Dutching over $N \backslash i$ to be profitable, it must hold that:

$$
\frac{1}{\alpha_{i}}>\pi(\boldsymbol{\alpha})
$$

in the time period where the Dutching portfolio is taken.

Proof. We note that if $N^{C}=N \backslash i$, then the Dutching payoff (Equation (3)) is:

$$
\left(\sum_{i \in N^{C}} \frac{1}{\alpha_{i}}\right)^{-1}=\frac{1}{\pi(\boldsymbol{\alpha})+1-\frac{1}{\alpha_{i}}}
$$

and solving for the expression being greater than one yields the result.

This creates an interesting link between the portfolio problem of Dutching and the naive probabilities. In other words, the naive probability of the outcome occurring must be greater than the excess naive probabilities on the market as a whole, or a profitable Dutching portfolio cannot be taken. We are therefore establishing a lower bound on the probability, which is the same as establishing an upper bound on $\alpha_{i}$. This might seem counter-intuitive, but the logic is that a Dutching portfolio replicating a lay bet takes place for all outcomes except the one that is desired to lay. Outcomes with low probabilities have high odds, and if these are too high, the balancing odds on the other outcomes may be too low to be able to create a viable Dutching portfolio. Consider the following example for illustration:

Example 10. Consider a market with $n=3$ possible outcomes and the odds $\alpha=(1.5,2,12)$, which implies $\pi(\boldsymbol{\alpha})=0.25$. Taking a replicating bet on the lay bet on Outcome 1 yields $(1 / 2+1 / 12)^{-1}=12 / 7>1$ by Equation 3, which means that if the is portfolio taken and Outcome 1 does not occur, the bettor makes a profit. This is consistent with Equation (13), as $1 / \alpha_{1}=1 / 1.5>\pi(\boldsymbol{\alpha})$. However, if it is attempted to replicate the lay bet on Outcome 3, the payoff is $(1 / 1.5+1 / 2)^{-1}=6 / 7<1$, which means that this portfolio is never profitable. This is also consistent with Equation (13), as $1 / \alpha_{3}=1 / 12<\pi(\boldsymbol{\alpha})$.

With this in mind, the general result for first taking a back bet and then replicating the lay position is as follows:

Proposition 6. Taking a back position and then closing it through replicating a lay bet through a Dutching portfolio over the back bets for $N \backslash i$ at a later stage, such that the same cash flow is guaranteed regardless of the outcome of the event, yields the return:

$$
\alpha_{i}\left(\frac{1}{\alpha_{i}^{\prime}}-\pi\left(\boldsymbol{\alpha}^{\prime}\right)\right)-1
$$

which means that a profit is made iff:

$$
\frac{1}{\alpha_{i}^{\prime}}-\frac{1}{\alpha_{i}}>\pi\left(\boldsymbol{\alpha}^{\prime}\right)
$$

Proof. See Appendix A.3

It is interesting that comparing the formula above to the most general case in Proposition 1, the naive probability $\frac{1}{\beta_{i}}$ is now replaced with the naive probability $\frac{1}{\alpha_{i}^{\prime}}-\pi\left(\boldsymbol{\alpha}^{\prime}\right)$, which is interpreted as the difference between the later naive back probability and the excess naive probabilities on the market as a whole. Similarly, the profit expression means that the difference in naive implied probability in the change over time must be greater than the back overround in the later time period. In other 
words, the changes in probabilities must be higher than the inefficiency in the market (created by the overround) in order to be able to hedge the position.

One can also take the trades in the opposite direction, first replicating the lay bet through the Dutching portfolio and then placing a back bet, hoping to profit from increases in odds. This results in the following:

Proposition 7. Taking a replicating position of a lay bet through taking a back bet portfolio over $N \backslash i$ and then closing it with a back bet at a later stage, such that the same cash flow is guaranteed regardless of the outcome of the event, yields the return

$$
\left(\pi(\boldsymbol{\alpha})+1-\frac{1}{\alpha_{i}}\right)^{-1} \times\left(1-\frac{1}{\alpha_{i}^{\prime}}\right)-1
$$

which means that a profit is made iff:

$$
\frac{1}{\alpha_{i}}-\frac{1}{\alpha_{i}^{\prime}}>\pi(\boldsymbol{\alpha})
$$

Proof. See Appendix A.4.

As is also the case for our previous results, the case of back and lay has the same profit expression, except with the time indexes switched out, as they should, though the profit formula is less intuitive due to the convoluted terms that go into it.

Note, however, that despite the more complicated expression, the intuitive explanation is the same as for Proposition 6. For Proposition 6, $\alpha_{i}$ represents the inverse of the implied naive probability of the back bet. The term $\frac{1}{\alpha_{i}^{\prime}}-\pi\left(\boldsymbol{\alpha}^{\prime}\right)$ represents the implied naive probability of the replicated back bet when expressed through the replicating formula. Similarly, for Proposition 7 , the term $\left(\pi(\boldsymbol{\alpha})+1-\frac{1}{\alpha_{i}}\right)^{-1}$ is the inverse of the naively implied probability through the replicating formula, while the term $1-\frac{1}{\alpha_{i}^{\prime}}$ is the implied naive probability of the back bet at the later stage. In this sense, the formulas have the same probabilistic interpretation as Propositions 1 and 2.

\section{Conclusions}

This paper provides the formalization for the concept of hedging on betting markets, taking an opposite bet to a previous one in order to make a profit or mitigating a loss through taking another position in the opposite direction. Two modifications to the simple case are treated: (i) the case where there is a betting exchange fee and (ii) the case where there is no option to lay in the market, and these are compared to the simple case.

Our results can be used in betting strategies for individuals, as they allow them to calculate profits and losses in theoretical future scenarios. However, from an academic perspective, they have the most use in the economic modeling of agents, as they allow for quantification of a profit for a bettor that does not wish to speculate on the outcome directly, but rather, the directions that the odds will move in, an aspect that is frequently neglected in the literature. In the same way that a model of a market of other financial instruments, such as options, does not give a complete picture unless the possibility to sell before maturity is taken into account, ignoring the possibilities to hedge in the manner described in this paper gives an incomplete understanding of pricing on betting markets. For the same reason, it would be interesting to investigate in an experimental setting where subjects that can bet on a market in multiple time periods are capable of detecting hedging opportunities and are able to exploit them for profit.

Author Contributions: Conceptualization, G.A.; methodology, G.A.; writing, original draft preparation, G.A.; writing, review and editing, G.A and D.C.; supervision, D.C. All authors read and agreed to the published version of the manuscript.

Funding: This research received no external funding.

Acknowledgments: The publication was sponsored by Pinnacle after the article was accepted. 
Conflicts of Interest: The authors declare no conflict of interest.

\section{Appendix A}

Appendix A.1. Proof of Proposition 2

Proof. First, the bettor risks one unit laying $i$, leaving him with an outstanding cash flow if any outcome except for $i$ occurs of $\frac{\beta_{i}}{\beta_{i}-1}$. He then takes a back bet on $i$ with the stake $\frac{1}{\alpha_{i}^{\prime}} \times \frac{\beta_{i}}{\beta_{i}-1}$, which gives a guaranteed cash flow of $\frac{\beta_{i}}{\beta_{i}-1}$ regardless of the outcome of the event. The return is then:

$$
\frac{\beta_{i}}{\beta_{i}-1}-\frac{1}{\alpha_{i}^{\prime}} \times \frac{\beta_{i}}{\beta_{i}-1}-1=\left(1-\frac{1}{\alpha_{i}^{\prime}}\right) /\left(1-\frac{1}{\beta_{i}}\right)-1
$$

regardless of the outcome of the event. Finding the condition for when there is a profit, we solve for the expression being greater than zero, ending up with $\alpha_{i}^{\prime}>\beta_{i}$.

Appendix A.2. Full Derivation of Proposition 3

For the back case, we take Proposition 1 and substitute Equations (9) and (10), giving us:

$$
\begin{aligned}
\frac{\alpha_{i}}{\beta_{i}^{\prime}}-1=\frac{1+(1-\tau)\left(a_{i}-1\right)}{1+\frac{b_{i}^{\prime}-1}{1-\tau}}-1 & >0 \\
(1-\tau)\left(a_{i}-1\right) & >\frac{b_{i}^{\prime}-1}{1-\tau} \\
\frac{a_{i}-1}{b_{i}^{\prime}-1} & >\frac{1}{(1-\tau)^{2}}
\end{aligned}
$$

For the lay case, we take Proposition 2 and substitute the same equations, giving us:

$$
\begin{aligned}
&\left(\frac{\beta_{i}}{\beta_{i}-1}\right) /\left(\frac{\alpha_{i}^{\prime}}{\alpha_{i}^{\prime}-1}\right)-1>0 \\
&\left(\frac{1+\frac{b_{i}-1}{1-\tau}}{1+\frac{b_{i}-1}{1-\tau}-1}\right) /\left(\frac{1+(1-\tau)\left(a_{i}^{\prime}-1\right)}{1+(1-\tau)\left(a_{i}^{\prime}-1\right)-1}\right)>1 \\
&\left(1+\frac{1-\tau}{b_{i}-1}\right)(1-\tau)\left(a_{i}^{\prime}-1\right)>1+(1-\tau)\left(a_{i}^{\prime}-1\right) \\
& \frac{a_{i}^{\prime}-1}{b_{i}-1}>\frac{1}{(1-\tau)^{2}}
\end{aligned}
$$

which shows the result.

\section{Appendix A.3. Proof of Proposition 6}

Proof. First, the bettor bets one unit $i$, leaving him with an outstanding positive cash flow of $\alpha_{i}$ if any outcome except $i$ occurs. Then, at a later stage, he takes a Dutching portfolio with a stake $\alpha_{i}\left(\sum_{i \in N \backslash i} \frac{1}{\alpha_{i}^{\prime}}\right)=\alpha_{i}\left(\pi\left(\boldsymbol{\alpha}^{\prime}\right)+1-\frac{1}{\alpha_{i}^{\prime}}\right)$, which gives a guaranteed cash flow of $\alpha_{i}$ regardless of the outcome of the event. The return is then:

$$
\alpha_{i}-\alpha_{i}\left(\pi\left(\boldsymbol{\alpha}^{\prime}\right)+1-\frac{1}{\alpha_{i}^{\prime}}\right)-1=\alpha_{i}\left(\frac{1}{\alpha_{i}^{\prime}}-\pi\left(\boldsymbol{\alpha}^{\prime}\right)\right)-1
$$

Finding the condition for when there is a profit, we easily solve for the return being greater than zero, ending up with $\frac{1}{\alpha_{i}^{\prime}}-\frac{1}{\alpha_{i}}>\pi\left(\boldsymbol{\alpha}^{\prime}\right)$. 


\section{Appendix A.4. Proof of Proposition 7}

Proof. First, the bettor bets one unit placing a Dutching portfolio over $N \backslash i$, leaving him with an outstanding cash flow of $\left(\sum_{j \in N \backslash i} \frac{1}{\alpha_{j}}\right)^{-1}=\left(\pi(\boldsymbol{\alpha})+1-\frac{1}{\alpha_{i}}\right)^{-1}$ if any outcome except for $i$ occurs. Then, at a later stage, he takes a back bet with the stake $\left(\pi(\boldsymbol{\alpha})+1-\frac{1}{\alpha_{i}}\right)^{-1} \times \frac{1}{\alpha_{i}^{\prime}}$, which gives a guaranteed cash flow of $\left(\pi(\boldsymbol{\alpha})+1-\frac{1}{\alpha_{i}}\right)^{-1}$ regardless of the outcome of the event. The return is then:

$$
\left(\pi(\boldsymbol{\alpha})+1-\frac{1}{\alpha_{i}}\right)^{-1}-\frac{\left(\pi(\boldsymbol{\alpha})+1-\frac{1}{\alpha_{i}}\right)^{-1}}{\alpha_{i}^{\prime}}-1=\left(\pi(\boldsymbol{\alpha})+1-\frac{1}{\alpha_{i}}\right)^{-1} /\left(\frac{\alpha_{i}^{\prime}}{\alpha_{i}^{\prime}-1}\right)-1
$$

\section{References}

Agrawal, Shipra, Nimrod Megiddo, and Benjamin Armbruster. 2010. Equilibrium in prediction markets with buyers and sellers. Economics Letters 109: 46-49. doi:10.1016/j.econlet.2010.08.017. [CrossRef]

Arrow, Kenneth J., Robert Forsythe, Michael Gorham, Robert Hahn, Robin Hanson, John O. Ledyard, Saul Levmore, Robert Litan, Paul Milgrom, Forrest D. Nelson, and et al. 2008. The promise of prediction markets. Science 320: 877-78. doi:10.1126/science.1157679. [CrossRef] [PubMed]

Axén, Gustav, and Dominic Cortis. 2019. Extending the price constraints of betting markets. Journal of Behavioral and Experimental Finance 23: 181-88. doi:10.1016/j.jbef.2019.07.001. [CrossRef]

Berg, Joyce E., Forrest D. Nelson, and Thomas A. Rietz. 2008. Prediction market accuracy in the long run. International Journal of Forecasting 24: 285-300. doi:10.1016/j.ijforecast.2008.03.007. [CrossRef]

Buhagiar, Ranier, Dominic Cortis, and Philip W. S. Newall. 2018. Why do some soccer bettors lose more money than others? Journal of Behavioral and Experimental Finance 18: 85-93. doi:10.1016/j.jbef.2018.01.010. [CrossRef]

Cain, Michael, David Law, and David Peel. 2000. The favourite-long shot bias and market efficiency in uk football betting. Scottish Journal of Political Economy 47: 25-36. [CrossRef]

Candila, Vincenzo, and Antonio Scognamillo. 2018. Estimating the implied probabilities in the tennis betting market: A new normalization procedure. International Journal of Sport Finance 13: 225-42.

Chatterjee, Subimal, and Satadruta Mookherjee. 2018. Valuing bets and hedges. Judgment and Decision Making 13: 509.

Chen, Yiling. 2005. Markets as an Information Aggregation Mechanism for Decision Support. Ph.D. thesis, The Pennsylvania State University, State College, PA, USA.

Cortis, Dominic. 2015. Expected values and variances in bookmaker payouts: A theoretical approach towards setting limits on odds. The Journal of Prediction Markets 9: 1-14.

Coulomb, Renaud, and Marc Sangnier. 2014. The impact of political majorities on firm value: Do electoral promises or friendship connections matter? Journal of Public Economics 115: 158-70. [CrossRef]

Dreber, Anna, Thomas Pfeiffer, Johan Almenberg, Siri Isaksson, Brad Wilson, Yiling Chen, Brian A. Nosek, and Magnus Johannesson. 2015. Using prediction markets to estimate the reproducibility of scientific research. Proceedings of the National Academy of Sciences 112: 15343-47. [CrossRef] [PubMed]

Flepp, Raphael, Stephan Nüesch, and Egon Franck. 2017. The liquidity advantage of the quote-driven market: Evidence from the betting industry. The Quarterly Review of Economics and Finance 64: 306-17. [CrossRef]

Franck, Egon, Erwin Verbeek, and Stephan Nüesch. 2010. Prediction accuracy of different market structures-Bookmakers versus a betting exchange. International Journal of Forecasting 26: 448-59. [CrossRef]

Frederick, Shane, Amanda Levis, Steven G Malliaris, and Andrew Meyer. 2018. Valuing bets and hedges: Implications for the construct of risk preference. Judgment and Decision Making 13: 501-8.

Kovalchik, Stephanie Ann. 2016. Searching for the goat of tennis win prediction. Journal of Quantitative Analysis in Sports 12: 127-38. [CrossRef]

Levitt, Steven D. 2004. Why are gambling markets organized so differently from financial markets? Economic Journal 114: 223-46. [CrossRef]

Manski, Charles F. 2006. Interpreting the predictions of prediction markets. Economics Letters 91: 425-29. [CrossRef] 
Newall, Philip W. S., and Dominic Cortis. 2019. High-stakes hedges are misunderstood too. A commentary on: "Valuing bets and hedges: Implications for the construct of risk preference". Judgment and Decision Making 14: 605.

Ottaviani, Marco, and Peter Norman Sørensen. 2005. Parimutuel versus Fixed-Odds Markets. Available online: http:/ / web.econ.ku.dk/sorensen/papers/pvfom.pdf (accessed on 30 June 2020).

Ottaviani, Marco, and Peter Norman Sørensen. 2009. Surprised by the parimutuel odds? American Economic Review 99: 2129-34. [CrossRef]

Peel, David. 2017. Wagering on more than one outcome in an event in cumulative prospect theory and rank dependent utility. Economics Letters 154: 45-47. [CrossRef]

Stark, Dudley, and Dominic Cortis. 2017. Balancing the book: Is it necessary and sufficient? The Journal of Gambling Business and Economics 11: 1-6.

Wolfers, Justin, and Eric Zitzewitz. 2006. Interpreting Prediction Market Prices as Probabilities. Technical Report. Cambridge: National Bureau of Economic Research.

(C) 2020 by the authors. Licensee MDPI, Basel, Switzerland. This article is an open access article distributed under the terms and conditions of the Creative Commons Attribution (CC BY) license (http:/ / creativecommons.org/licenses/by/4.0/). 